\title{
Erratum
}

\section{Studying links via closed braids IV: composite links and split links}

\author{
Joan S. Birman ${ }^{1}$, William W. Menasco ${ }^{2}$ \\ 1 Department of Mathematics, Barnard College \& Columbia University, 2990 Broadway, \\ NY 10027, USA (e-mail: jb@math. columbia. edu) \\ 2 Department of Mathematics, SUNY at Buffalo, Buffalo, NY 14222, USA \\ (e-mail: menasco@tait.math.buffalo.edu)
}

Invent. math. 102, 115-139 (1990)

Oblatum 7-XI-2003 \& 10-VIII-2004

Published online: 20 January 2005 - (c) Springer-Verlag 2005

The purpose of this erratum is to fill a gap in the proof of the 'Composite Braid Theorem' in the manuscript Studying Links Via Closed Braids IV: Composite Links and Split Links, Invent. Math. 102, Fasc. 1, 115-139 (1990). The statement of the theorem is unchanged. The gap occurs on p. 135 , lines $13^{-}$to $11^{-}$, where we fail to consider the case:

$V_{2}=4, V_{4}>0, V_{j}=0$ if $j \neq 2,4$, and all 4 vertices of valence 2 are bad.

At the end of this Erratum we make some brief remarks on the literature, as it evolved during the 14 years between the publication of [3] and the submission of this Erratum.

See Fig. 1 below for an example which illustrates that the missing case can occur. Ivan Dynnikov discovered it when he was working on his manuscript [7], where he established two theorems about arc presentations of links which are similar to the two theorems that we had proved for closed braid presentations in [3]. His proof was a modification of our proof to new geometry, and in the course of his work he realized there was a gap. We thank him for pointing it out to us.

To handle the missing case, we need to change the definition of a good vertex, on p. 135, lines 23-24. A vertex $v$ in the foliation of our 2-sphere is good if every non-singular leaf adjacent to $v$ has empty intersection with $\mathbf{K}$. (As stated, we are always assuming that $\mathbf{K}$ intersects the foliation of $\mathbf{Y}$ away 


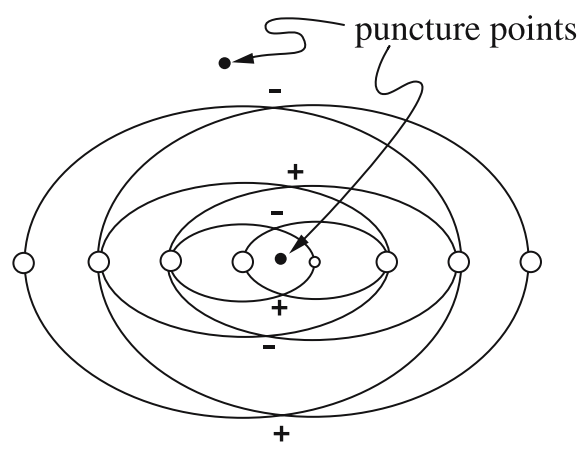

Fig. 1. A tiling of the 2-sphere $\mathbf{Y}$ with $V_{2}=V_{4}=4, V_{j}=0$ if $j \neq 2,4$, and all vertices of valence 2 are bad

from singular leaves.) With this change, the definition of a good tile is no longer needed or used.

We now discuss how to handle the missing case, by showing that there is a new choice of disc fibers for the fibration which, in effect, "moves one of the punctures out of a bad region', thereby making it good. After that we will be able to reduce the complexity. The proof adapts an argument used at a later point in [3] to the situation that we overlooked.

A disc region $R \subset \mathbf{Y}$ is a sub-disc of $\mathbf{Y}$ whose boundary is contained in the union of two singular leaves and whose interior is foliated by a family of $b$-arcs. See Fig. 2 below and Fig. 6 of [3].

Lemma 1. Let $R$ be a disc region in the tiling of $\mathbf{Y}$, with $R \cap \mathbf{K}$ a single point, also $\partial R \subset l_{+} \cup l_{-}$, where $l_{ \pm}$is a singular leaf whose associated singularity has sign \pm . Then there exists a new choice of disc fibers which leaves the the tiling of $\mathbf{Y}$ unchanged, but after the change $R \cap \mathbf{K}=\emptyset$. Moreover, if $\mathbf{Y}$ is oriented, and if $R \cap \mathbf{K}$ is a positive (resp. negative) puncture then the change of fibration corresponds to an isotopy of $\mathbf{K}$ across $l_{+}\left(\right.$resp. $\left.l_{-}\right)$. As illustrated in Fig. 2, there are two possible isotopies.

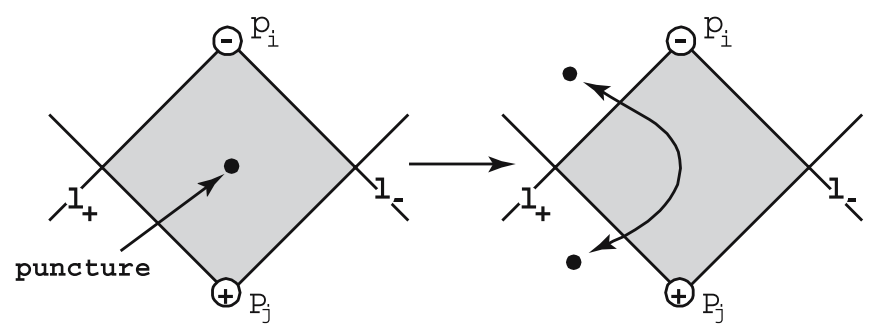

Fig. 2. We illustrate the two ways that one of the puncture points can be moved past the singular leaf. Both ways are realizable 
Proof. To simplify notation we consider a special case, assuming that:

- The negative singularity of $R$, the $b$-arcs in $R$, and the positive singularity of $R$ occur in that order in the fibration.

- The puncture point $R \cup \mathbf{K}$ is positive.

We will establish that the order of the positive puncture and the positive singularity in the fibration can be interchanged by re-choosing the disc fibers. The special case is in fact the general case because the other order and parity possibilities can be realized by reversing the orientation of $\mathbf{Y}$ and/or reversing the orientation of the fibration.

Referring to sketch (a) in Fig. 3, we depict the $H_{\theta}$-sequence from the instant just before the positive puncture point to the instant right after the positive singularity. Observe that the $b$-arcs split $H_{\theta}$ into two subdisc $\Delta_{\theta}^{1} \cup \Delta_{\theta}^{2}=H_{\theta}$ where $\Delta_{\theta}^{1}$ is on the negative side of the $b$-arcs and $\Delta_{\theta}^{2}$ is on the positive of the $b$-arcs. Thus the events - singularities and braiding - in the fibration that occur in $\Delta_{\theta}^{1}$ are independent of the events that occur in $\Delta_{\theta}^{2}$. This allows us to alter the $H_{\theta}$-sequence. In particular, prior to the instant when $\mathbf{K}$ punctures $\mathbf{Y}$ we can "push" forward the fibration on the $\Delta_{\theta}^{1}$ side until precisely after the occurrence of the positive singularity. We then allow $\mathbf{K}$ to puncture $\mathbf{Y}$. The "dot" in $\Delta_{\theta}^{1} \cap \mathbf{K} \subset H_{\theta}$ that is used in (a) of Fig. 3 to create the puncture can be placed close to the $b$-arcs of $R$ away from the other events as we do this "push". We have a choice of which side of the positive singularity we want the "dot"; thus, when the dot finally passes through $\mathbf{Y}$, it can be on either side of the singularity. The sketches in (b) of Fig. 3 illustrates the resulting change of fibration. Figure 4(a) illustrates the (two possible) 3-dimensional geometric realization of the $H_{\theta}$-sequence of Fig. 3. (For completeness we also have Fig. 4(b) which illustrates a possible obstruction to a change of fibration when the parity of the puncture and singularity disagree.) All of the other events are combinatorially unaltered by this new choice of disc fibers, therefore the foliation of the $\mathbf{Y}$ is unchanged. II
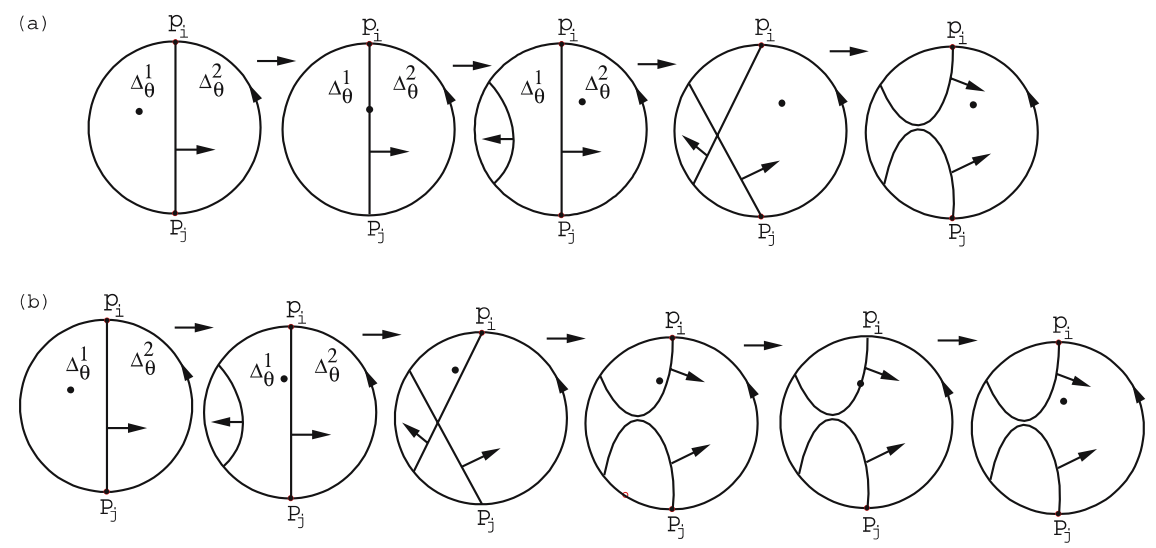

Fig. 3. The two $H_{\theta}$ sequences in the proof of Lemma 1 


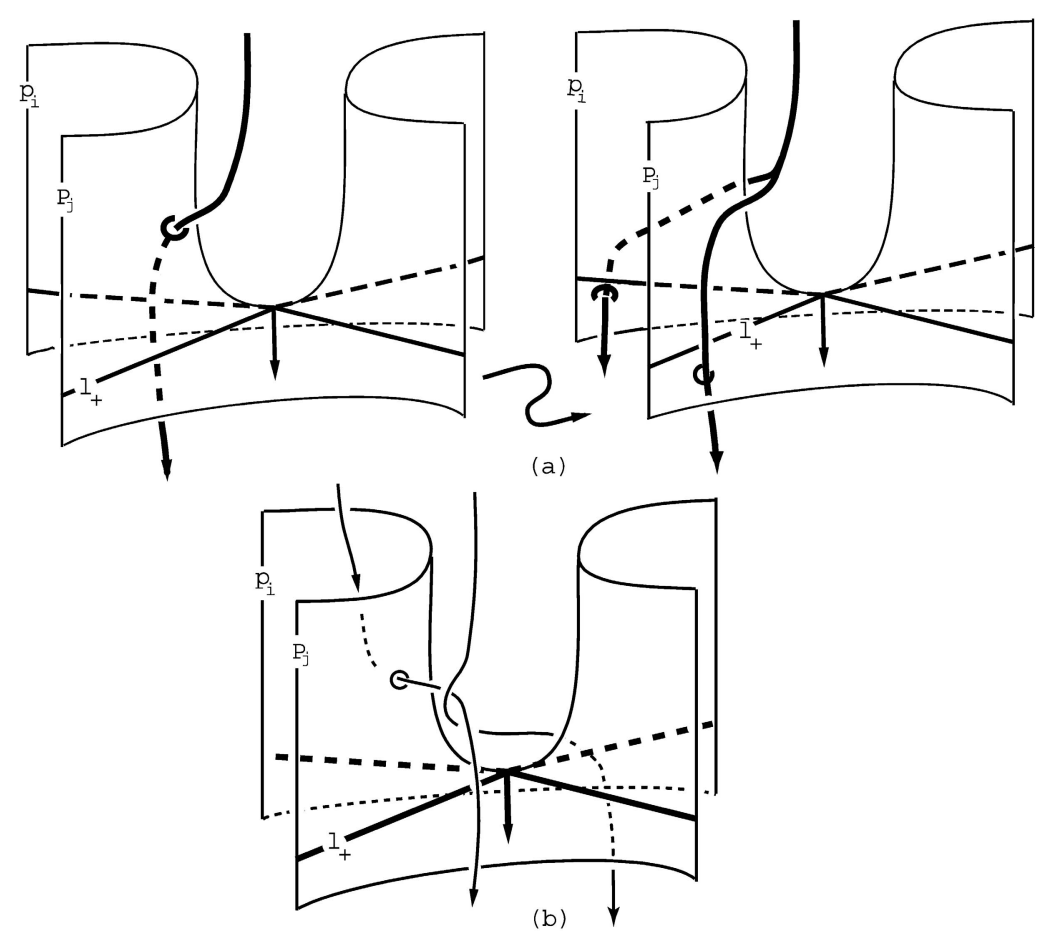

Fig. 4. a The geometric realization of the change in fibration in Fig. 2. b We illustrate a possible obstruction to a change of fibration when the parity of the puncture and singularity disagree

Lemma 2. See Fig. 13 of [1]. Let link $(p)=R_{1} \cup R_{2}$ be the link neighborhood of a valence two vertex $p$ in the tiling of the 2-sphere $\mathbf{Y}$ which realizes the connected sum where $R_{1} \& R_{2}$ are disc regions. Assume that $\left|R_{i} \cap \mathbf{K}\right|<2, i=1,2$. Then after the change of fibration of Lemma 1 we can perform an exchange move on $\mathbf{K}$ and an isotopy of $\mathbf{Y}$ which eliminates $p$.

Proof. Suppose that $R_{1}$ is a disc region in the link of vertex $p$ of valence two. Referring to Fig. 13 of [1], we have two disc regions containing $b$-arcs, with $\left\{t_{2}, t_{3}, t_{4}\right\}$ corresponding to $R_{1}$ and $\left\{t_{6}, t_{7}, t_{8}\right\}$ respectively corresponding to $R_{2}$. Since $\operatorname{link}(p)$ has both a positive and a negative singularity, the disc region $R_{1}$ also has both positive and negative singular points in its boundary. By assumption $R_{1}$ has at most one puncture point. We apply Lemma 1 to move the puncture point out of $R_{1}$ (but not into $R_{2}$ ). This is possible since as illustrated in Fig. 2 there are two possible change of fibration isotopies and we can choose which side of a singular point the puncture passes by. Similarly, for $R_{2}$. After our application of the lemma making $\operatorname{link}(p)$ a good disc we know we can apply an exchange move to eliminate the valence two vertex. ||

Lemmas 1 and 2 handle the missing case in the proof of the Composite Braid Theorem of [1]. ॥ 
In closing, we use this opportunity to correct an error in the signs of certain crossings in Fig. 22 of [3]. Each of the two crossings of the strand that is labeled " $\mathrm{k}$ " (resp. the strand that is labeled "m") with the axis A should be reversed.

Remarks on the literature: For the benefit of the reader, we note that during the 14 years between the publication of [3] and the submission of this Erratum, there have been many papers published which use the braid foliation techniques introduced in [3], for example [4], [5], [6], [7], [8] and [9]. For that reason, 8 years after the manuscript [3] appeared, the first author and E. Finkelstein wrote a review article on braid foliations [2]. It contains, in particular, careful proofs of the validity of the change in fibration and the exchange move, both of which first appeared in [3]. The interested reader may find it helpful to consult [2] if he/she is interested in learning the basic techniques in braid foliation theory.

In a different direction, readers have pointed out to us the similarity between many of our techniques and those of D. Bennequin in [1]. As one example, the technique that we use in the proof of Lemma 3 of [3] to reduce the valence of the two middle vertices in the sketches labled (c) in Fig. 18 of [3] is similar to the argument that is given in the proof of Theorem 6 of [1], in the (unnumbered) Lemma that appears in the course of the proof of Theorem 6 of [1]. Those similarities puzzled us greatly for many years, because Bennequin was studying the so-called characteristic foliation of a surface in 3-space supplied with a contact struture, and not the braid foliation of the same surface in 3-space supplied with a braid structure. When we first learned of such similarities we convinced ourselves that the characteristic foliation and the braid foliation were genuinely different, and that most of the extensive literature resulting from Bennequin's foundational work [1] simply did not apply to braid foliations. After recent conversations with Ivan Dynnikov, we finally understood the explanation: the essential hypotheses needed to prove particular overlapping technical lemmas are equally valid in the setting of braid foliations and characteristic foliations. Bennequin did not prove any of the theorems in [3] or our subsequent papers based upon braid foliation techniques. Moreover, it seems to us that any application of results worked out in either setting to the other setting would require a great deal of careful checking, because the underlying setting, terminology, basic definitions and references are all distinct.

\section{References}

1. Bennequin, D.: Entrelacements et equations de Pfaff. Astérisque 107-108, 87-161 (1983)

2. Birman, J.S., Finkelstein, E.: Studying surfaces va closed braids. J. Knot Theory Ramifications 7, 267-334 (1998)

3. Birman, J.S., Menasco, W.W.: Studying Links Via Closed Braids IV: Composite Links and Split Links. Invent. Math. 102, 115-139 (1990)

4. Birman, J.S., Menasco, W.W.: Studying Links Via Closed Braids V: Closed Braid Representatives of the Unlink. Trans. Am. Math. Soc. 329, 585-606 (1992) 
5. Birman, J.S., Menasco, W.W.: Studying Links Via Closed Braids VI: “A Non-Finiteness Theorem" (with W. Menasco). Pac. J. Math. 156, 265-285 (1992)

6. Cromwell, P.: Embedding knots and links in an open book I: basic properties. Topology Appl. 64, 37-58 (1995)

7. Dynnikov, I.A.: Arc presentations of links. Monotonic simplifications. Preprint, arXiv:math.GT/0208153 v2

8. Fintushel, R., Stern, R.: Symplectic surfaces in a fixed homology class. J. Differ. Geom. 52, 203-222 (1999)

9. Matsuda, H.: Complements of hyperbolic knots of braid index four contain no closed embedded totally geodesic surfaces. Topology Appl. 119, 1-15 (2002) 